\title{
Familia como espacio de resiliencia en las personas con capacidades diferentes de la Facultad de Ciencias Humanísticas y Sociales
}

Family as a space of resilience in people with different capabilities of the faculty of humanistic and social sciences

Mayra Ivonne Villamar Delgado' Tatiana Katiuska Moreira Chica 2

RECIBIDO: 9 DE MAYO DE 2020

ACEPTADO: 15 DE JUNIO DE 2020

\section{RESUMEN}

Esta investigación relaciona conceptos de resiliencia y discapacidad en un conjunto de criterios que involucra a la familia como primera organización social, por ello, el objetivo consistió en identificar a la familia como espacio de resiliencia en las personas con capacidades diferentes de la Facultad de Ciencias Humanísticas y Sociales. Para lo cual se utilizó una metodología de tipo no experimental, se aplicó el método bibliográfico, inductivo, deductivo, su ejecución fue de nivel descriptivo análisis y síntesis, también se aplicó la técnica de la entrevista y como instrumento la

\footnotetext{
I Egresada de Trabajo Social, Universidad Técnica de Manabí, Ecuador; mvillamar1971@utm.edu.ec, ORCID: https://orcid.org/0000-0003-0703-998X

Google Scholar: https://scholar.google.es/citations?hl=es\&user=ULhYTWUAAAAJ

2 Magister en Educación y Desarrollo Social, Licenciada en Trabajo Social, Docente, Universidad Técnica de Manabí, Ecuador; tmoreira@utm.edu.ec, ORCID: https://orcid.org/0000-0001-5655-4721

Google Scholar: https://scholar.google.es/citations?hl=es\&user=-ZK5jkIAAAAJ
} 


\section{8}

escala de resiliencia mexicana RESIM de los autores Palomar y Gómez 2010, la cual fue dirigida a una población y muestra de 5 familias con discapacidad. Como resultado se evidencia que los integrantes de estas familias disponen de un estándar medio y alto en la comprensión de resiliencia, esto se relaciona con los tres elementos analizados, es decir que el valor que se le otorga a la resiliencia de carácter personal, familiar y social les permite mirar positivamente en la realidad en la que viven. Como conclusión se destaca la construcción de la resiliencia en la familia que tienen personas con capacidades diferentes, donde existe una interrelación personal, familiar y comunitaria lo que lleva a un punto de intersección para fortalecer a está de manera trasversal, es decir, que se hace uso de los recursos personales.

Palabras clave: discapacidad, resiliencia, familia, Facultad de Ciencias Humanísticas y Sociales

\section{ABSTRACT}

This research relates concepts of resilience and disability in a set of criteria that involves the family as the first social organization, therefore, it aims to identify the family as a space of resilience in people with different abilities from the faculty of humanistic sciences and social. For which a non-experimental type methodology was used, with an inductive-deductive approach, it was based on the descriptive, bibliographic method. The interview technique was used and as an instrument the interview guide and the Mexican RESIM resilience scale applied to a population and sample of 5 families of students of the faculty with families with disabilities. As a result, it is evident that the members of these families have a medium high and high standard in understanding resilience, this is related to the three elements analyzed, that is, the value that is given to resilience of a personal, family nature. and social allows them to look positively at the reality in which they live. To conclude The construction of resilience in the family that has people with different capacities, has a personal, family and community interrelation which leads to a point of intersection to strengthen this in a transversal way, that is, that use is made of the personal resources.

Keywords: disability, resilience, family, Faculty of Humanistic Sciences 


\section{Introducción}

La familia se la puede considerar como el centro de integración entre personas, donde cada quien es acogido en su seno de modo integral, debido a que está constituida por una serie de aspectos biológicos, psicológicos, sociales y trascendentales, ésta se inicia con la formación de una pareja y que, en realidad, la evolución de esas relaciones de pareja constituye el eje medular de la vida familiar. Según (Valladáres, 2017) "La familia es el referente más relevante, su papel es esencial en el proceso de cuidado, crianza y educación de los hijos, es el entorno más adecuado para que las personas se desarrollen de manera integral". Es decir que esta institución permite concienciar aspectos de apoyo e integración conjunta entre las diferencias y dificultades que puedan presentar sus integrantes, en una investigación realizada por (Puerta, 2017) menciona que "la familia puede ser un ámbito potencialmente generador de resiliencia, factor fundamental al cual necesitan prestar la mayor atención los puericultores científicos, llamados a orientar y acompañar a las familias en los procesos de crianza de sus hijos". De acuerdo a esta investigación, en la familia se puede generar resiliencia lo que permite superar dificultades y orientar los objetivos que se direccionan al éxito de todos sus integrantes.

Según (Gomez y Kotliarenco, 2010) "La resiliencia familiar se define como el conjunto de procesos de reorganización de significados y comportamientos que activa una familia sometida a estrés para recuperar y mantener niveles óptimos de funcionamiento y bienestar, equilibrando sus recursos y necesidades familiares". En este aspecto el equilibrio es fundamental para alcanzar el bienestar en las personas, sobre todo cuando existe alguna condición como la discapacidad, que limita ciertas situaciones en la persona quien la posee.

La forma en que nos expresamos define y difunde maneras de ver el mundo y de entender a las personas Por otra parte, al referirnos a una persona en particular, lo correcto es llamarla por su nombre, como a cualquier otro individuo. En caso de que debamos referirnos a la discapacidad, la expresión correcta es la de persona con discapacidad. En primer lugar, hablamos de las personas y en segundo término de la discapacidad como una de sus características. Por ello, el objetivo de esta investigación consiste en identificar a la familia como espacio de resiliencia en las 
personas con capacidades diferentes de la Facultad de Ciencias Humanísticas y Sociales.

\section{Desarrollo}

El enfoque de la resiliencia familiar (Gómez y Kotliarenco, 2010) plantea que existen factores protectores lo cual apunta a la función de "escudo" que ciertas variables cumplen sobre el funcionamiento familiar para mantenerlo saludable y competente bajo condiciones de estrés: por ejemplo, las celebraciones familiares, el tiempo compartido o las rutinas y tradiciones familiares. Por lo tanto, es importante considerar que se debe de dar un proceso de adaptación, el cual en cada familia es diverso. De modo que, si se parte de esta realidad, se establecen espacios y tiempos para el andamiaje de la adaptación, se construyen estrategias las cuales generan formas de afrontar dicha situación, todo este proceso tiene de fondo la edificación de un proceso resiliente.

\section{Resiliencia Familiar}

El abordaje de la resiliencia en familias entre otros aspectos, identifica los procesos importantes para superar una crisis que pudiera alterar la armonía de sus integrantes, pero este proceso implica factores que involucran disciplinas, tal como lo señala (Truffino, 2009) quien expresa que "sigue siendo un concepto poco evidente y no claramente definido debido a la existencia de múltiples factores implicados y a su desarrollo desde disciplinas tan diversas como la medicina, la psicología, la educación, el trabajo social o la epidemiología".es decir que al implicar una base de ciencias, su compresión se define de manera confusa desde un punto de abordaje básico. Sin embargo, al referir directamente el aspecto social, (Oriol-Bosch, 2012) manifiesta:

La resiliencia social depende de la confianza mutua y de la fortaleza de los vínculos en el grupo y expresa su capacidad de absorber las presiones y estabilizarse rápidamente. Las familias y las comunidades han sido objeto de estudios sobre los factores de resiliencia en condiciones de adversidad, y se han encontrado elementos materiales, culturales y políticos capaces de influir en sus 
consecuencias. El apoyo comunitario es un elemento efectivo para el desarrollo de la resiliencia individual y grupal.

La resiliencia representa el resultado de anhelos para mantener el equilibrio ante dificultades que interrumpan la tranquilidad de la familia, se basa en un esquema de condiciones que permiten mejorar el estado emocional y afectivo, basados en la reciprocidad y el entendimiento que influye en mejoras familiares de las situaciones. No obstante, esto implica una serie de procedimientos que se implementan a medida que trascurre el tiempo, (Martínez, 2012) expresa que:

Estas dos situaciones, es decir, tener una discapacidad adquirida desde el nacimiento o tenerla sobrevenida a lo largo de la vida, no van a variar el hecho de tener una discapacidad, ni tampoco la multitud de barreras tanto físicas como mentales a las que desde la discapacidad hay que solventar, pero la capacidad para afrontarlas y sobreponerse si puede ser diferente.

Las personas con capacidades diferentes son consideradas como ser humano que presente temporal o permanentemente una limitación, por pérdida o disminución de sus facultades físicas, intelectuales o sensoriales, que limite realizar sus actividades connaturales. Esto no puede representar una limitación social, por ello involucra al desarrollo social inclusivo, el cual se fundamenta en el diseño e implementación de acciones y políticas públicas para todos los ecuatorianos, para que puedan acceder a la igualdad de oportunidades, independientemente de su estatus social, género, edad, condición física y mental, etnia, religión entre otras.

En la constitución de la republica del Ecuador (2008) en el artículo 46 numeral 3 menciona sobre la "atención preferente para la plena integración social de quienes tengan discapacidad. El Estado garantizará su incorporación en el sistema de educación regular y en la sociedad". Es decir, que no se puede condicionar a una persona con discapacidad, para ingresar al sistema de educación, en este contexto, el artículo 47 establece que "El Estado garantizará políticas de prevención de las discapacidades y, de manera conjunta con la sociedad y la familia, procurará la equiparación de oportunidades para las personas con discapacidad y su integración social". Es indispensable que las personas con discapacidad gocen de una inclusión 


\section{2}

que permita igualdad de oportunidades y para sus familiares garanticen una tranquilidad emocional, al sentir que éstas son útiles en el aspecto social, educativo, laboral y económico para el país.

La persona con capacidades diferentes que cursan una carrera universitaria gozan de una inclusión educativa al sentir las adaptaciones de las instalaciones a su condición, tal como menciona la (UNESCO, 2017) "la elaboración e implementación de políticas, programas y prácticas que promueven la inclusión en el ámbito educativo, con miras a garantizar la igualdad de oportunidades de las personas discapacitadas en materia de educación". Cabe indicar que la adaptación de las instituciones, no garantizan una inclusión directa, ya que existe el proceso para la integración de estudiantes a las carreras universitarias.

\section{Metodología}

En esta investigación es de tipo no experimental, se utilizó el método bibliográfico, inductivo, deductivo, su ejecución fue de nivel descriptivo análisis y síntesis, lo cual permitió obtener información relevante del objeto de estudio se aplicó la técnica de la entrevista y como instrumento la escala de resiliencia mexicana RESIM de los autores Palomar y Gómez 2010, la cual fue dirigida a una población y muestra de 5 familias con discapacidad de estudiantes de la Facultad de Ciencias Humanísticas y Sociales de la Universidad Técnica de Manabí . La información se obtuvo a partir de la entrevista estructurada con preguntas cerradas la cual se realizó de forma específica. 


\section{Resultados}

\section{Tabla 1}

Características de los casos

\begin{tabular}{|c|c|c|c|c|c|}
\hline Tipos de Familias & $\begin{array}{c}\text { Familia } 1 \\
\text { Reconstruida }\end{array}$ & $\begin{array}{c}\text { Familia } 2 \\
\text { Monoparental }\end{array}$ & $\begin{array}{c}\text { Familia } 3 \\
\text { Monoparental }\end{array}$ & $\begin{array}{c}\text { Familia } 4 \\
\text { Monoparental }\end{array}$ & $\begin{array}{c}\text { Familia } 5 \\
\text { Monoparenta }\end{array}$ \\
\hline Estado civil & Casados & Separados & separados & casados & divorciados \\
\hline Nivel educativo & Básico & licenciatura & licenciatura & Masterado & Licenciatura \\
\hline $\begin{array}{l}\text { Estatus } \\
\text { socioeconómico }\end{array}$ & bajo & medio & Medio & Medio alto & medio \\
\hline $\begin{array}{l}\text { Edad de la } \\
\text { persona con } \\
\text { discapacidad }\end{array}$ & 14 & 11 & 13 & 10 & 7 \\
\hline $\begin{array}{l}\text { Sexo de la } \\
\text { persona con } \\
\text { discapacidad }\end{array}$ & hombre & hombre & Hombre & mujer & mujer \\
\hline $\begin{array}{c}\text { Diagnóstico de la } \\
\text { persona con } \\
\text { discapacidad }\end{array}$ & $\begin{array}{c}\text { Daño } \\
\text { neurológico } \\
\text { con } \\
\text { discapacidad } \\
\text { motora }\end{array}$ & $\begin{array}{l}\text { Trastorno } \\
\text { general del } \\
\text { desarrollo }\end{array}$ & $\begin{array}{l}\text { Hidrocefalia } \\
\text { compensada }\end{array}$ & $\begin{array}{c}\text { Daño } \\
\text { neurológico }\end{array}$ & $\begin{array}{c}\text { Daño } \\
\text { neurológico }\end{array}$ \\
\hline
\end{tabular}

Nota. Familias con discapacidad de estudiantes de la Facultad de Ciencias Humanísticas y Sociales 2020.

Para la obtención de estos resultados se identifica una numeración a las familias entrevistadas las cuales se enumero del 1 al 5, cabe indicar que esta entrevista se realizó al representante familiar. De manera oportuna se aplico la escala de resiliencia (RESIM) (Palomar \& Gomez, 2010) la cual valora la resiliencia desde diversos aspectos, ésta busca obtener una aproximación a esta concepción en cada participante

La escala se distribuye en cinco dimensiones: fortaleza y confianza en sí mismo; competencia social; apoyo familiar; apoyo social; y estructura. Sin embargo, en esta investigación se utilizaron solo 3: Resiliencia Personal (Confianza en sí mismo); Resiliencia Familiar (Familia); Resiliencia Comunitaria (Apoyo social). Estas fueron considerarlos adecuados al objetivo inicial, estos se acercan al carácter personal, familiar y comunitario. Es importante mencionar que en esta se organizó a los 


\section{4}

participantes según su rol de la siguiente forma: M (madre), P (padre), HM (hermano/a mayor), Hm (hermano/a menor), Hi (hermano paterno/materno) T (tía o tío)

En esta sección se concentra en conocer a los participantes, obtener información, para ello fue necesario los siguientes datos de cada familia y sus integrantes, para lo cual se obtuvieron los siguientes resultados.

\section{Tabla 2}

Respuestas de cada Familia y sus integrantes

\begin{tabular}{|c|c|c|c|c|c|c|c|c|c|c|c|c|}
\hline \multirow[t]{2}{*}{ Familia } & \multicolumn{4}{|c|}{ Familia 1} & \multicolumn{2}{|c|}{ Familia 2} & \multicolumn{2}{|c|}{ Familia 3} & \multicolumn{2}{|c|}{ Familia 4} & \multicolumn{2}{|c|}{ Familia 5} \\
\hline & $M$ & $P$ & $\mathrm{MH}$ & $\mathrm{HI}$ & $M$ & $T$ & $M$ & $\mathrm{Hi}$ & $M$ & HM & $M$ & $\mathrm{HM}$ \\
\hline $\begin{array}{l}\text { Escala de } \\
\text { resiliencia total }\end{array}$ & $\begin{array}{c}\text { Media } \\
\text { Alta }\end{array}$ & Alta & Alta & Alta & Alta & $\begin{array}{l}\text { Media } \\
\text { Alta }\end{array}$ & $\begin{array}{c}\text { Media } \\
\text { Alta }\end{array}$ & Alta & Alta & Alta & Alta & Alta \\
\hline $\begin{array}{l}\text { Resiliencia } \\
\text { personal }\end{array}$ & $\begin{array}{c}\text { Media } \\
\text { Alta }\end{array}$ & Alta & Alta & Alta & Alta & $\begin{array}{c}\text { Media } \\
\text { Alta }\end{array}$ & $\begin{array}{c}\text { Media } \\
\text { Alta }\end{array}$ & Alta & Alta & Alta & Alta & Alta \\
\hline $\begin{array}{l}\text { Resiliencia } \\
\text { familiar }\end{array}$ & $\begin{array}{c}\text { Media } \\
\text { Alta }\end{array}$ & Alta & Alta & Alta & Alta & $\begin{array}{l}\text { Media } \\
\text { Alta }\end{array}$ & $\begin{array}{c}\text { Media } \\
\text { Alta }\end{array}$ & Alta & Alta & Alta & Alta & Alta \\
\hline $\begin{array}{l}\text { Resiliencia } \\
\text { comunitaria } \\
\text { (apoyo social) }\end{array}$ & $\begin{array}{c}\text { Media } \\
\text { Alta }\end{array}$ & Alta & Alta & Alta & Alta & $\begin{array}{c}\text { Media } \\
\text { Alta }\end{array}$ & $\begin{array}{c}\text { Media } \\
\text { Alta }\end{array}$ & Alta & Alta & Alta & Alta & Alta \\
\hline
\end{tabular}

Nota. Familia de estudiantes con integrantes con discapacidad de la Facultad de Ciencias Humanísticas y Sociales 2020.

Estos resultados permitieron observar que los integrantes de estas familias disponen de un estándar medio alto y alto en la comprensión de resiliencia, esto se relaciona con los tres elementos analizados, es decir que el valor que se le otorga a la resiliencia de carácter personal, familiar y social les permite mirar positivamente en la realidad en la que viven, esto genera entre ellos fortaleza para el diario vivir. 


\section{Discusión}

La información que se obtuvo de las familias objeto de estudio dejó en evidencia que estas cuentan con una alta resiliencia para superar obstáculos, esto implica que han logrado adaptarse a las circunstancias y afrontan de forma positiva los problemas que se presenta, tal como menciona (Vargas, 2018) el cual afirma:

La resiliencia como constructo social plantea un panorama conceptual confuso y diverso el cual incluye su visualización como cualidad individual, como rasgo colectivo, o como proceso dinámico, entre otros, con el común denominador de la adaptación que se genera ante la vivencia de una situación retadora.

A modo particular la resiliencia enfoca la cualidad de las personas para afrontar una situación, que viabiliza una ruta de solución que se empodere del ser humano como factor de cambio ante situaciones agudas, es decir, da la respuesta a un acontecimiento, toma la potestad de estabilizar esa situación en bienestar con tranquilidad equivalente a un equilibrio dinámico con factores asociados a la tranquilidad. Así lo mencionan (Espinoza Alveal y Matamala Yáñez, 2012) quienes afirman:

La resiliencia está asociada con factores protectores y factores de riesgo, entendiéndose por los primeros aquellas condiciones o contextos que promueven el desarrollo de las personas o grupos, producto de que intervienen como amortiguadores ante situaciones complejas o de riesgo que podrían causar un gran impacto en el individuo e influir negativamente, impidiendo su progreso.

En relación al contexto de las personas o grupos, se evidencia en el grupo familiar entrevistado en el cual su escala de resiliencia es alta, evidenciando que manejan la situaciones complejas, tomando las mismas con absoluta tranquilidad, evitando colapso emocional, es importante mencionar lo expuesto por (Benetti, 2010) quien manifiesta "que Los factores de protección identificados en las familias estaban relacionados con las características personales de sus miembros, tales como: talentos especiales, valoración de los estudios, religiosidad, lazos comunitarios y determinación en cambiar la situación financiera". Esto se evidencia de manera 


\section{6}

proporcional en los resultados de la tabla 2 en donde se caracterizan los resultados sobre resiliencia en los grupos familiares con el valor de muy alta

En los análisis sobre el elemento de apoyo social como aspectos de una resiliencia comunitaria, se halló que en las familias el contar con una red de apoyo era primordial en la familia reconstruida, estos básicamente hacen énfasis en (iglesias, escuelas, centros de reuniones sociales entre otros) estos enlazan la construcción de resiliencia en las familias que tienen la discapacidad en hijos o hermano. Se menciona que esta red de apoyo se la consolida con fuente de solidaridad la cual genera vínculos emocionales, reconocimiento, acompañado de valores que se experimentan en comunidad.

\section{Conclusión}

Es importante mencionar que la resiliencia en la familia se lo considera como un proceso complejo y sistémico el cual requiere una serie de condiciones que involucran aspectos tanto personales, familiares y comunitarios lo cual tiene como base una característica de cada individuo.

La construcción de la resiliencia en la familia que tiene personas con capacidades diferentes, tiene una interrelación personal, familiar y comunitaria lo que lleva a un punto de intersección para fortalecer está de manera trasversal, es decir, que se hace uso de los recursos personales, se apoya en el grupo familiar para así, empoderarse de la realidad en relación con el exterior en el cual se desarrolla la comunidad

En relación a la entrevista realizada a 5 familias, de manera amplia se ratifica la presencia de fortalezas de una manera útil para mantener el equilibrio emocional, pese a situaciones complejas que se genera al existir un familiar con capacidades diferentes, lo que no es una limitación para continuar con las actividades cotidianas. 


\section{Referencias}

Benetti, S. P. (22 de mayo de 2010). Universidad Santo Tomás . Obtenido de https://revistas.usantotomas.edu.co/index.php/diversitas/article/view/141/20 4

Ecuador, C. d. (20 de Octubre de 2008). Oas. Obtenido de https://www.oas.org/juridico/pdfs/mesicic4_ecu_const.pdf

Espinoza Alveal, M. A., y Matamala Yáñez, A. E. (2012). Universidad del Bio Bio. Obtenido de http://repobib.ubiobio.cl/jspui/bitstream/123456789/1437/1/Espinoza\%20Alve al\%2C\%20Maria\%20Aurora.pdf

Gómez, E., y Kotliarenco, M. (2010). Resiliencia Familiar: un enfoque de investigación e intervención con familias multiproblemáticas. Revista de Psicología, 107108.

Gomez, E., y Kotliarenco, M. A. (13 de Julio de 2010). Resiliencia Familiar: un enfoque de investigación e intervención con familias multiproblemáticas. Revista de Psicología de la Universidad de Chile, 19(2), 103. Recuperado el 29 de Marzo de 2020, de https://www.redalyc.org/articulo.oa? id=26416966005

Martínez, R. S. (Julio de 2012). Universidad de Valencia. Recuperado el 29 de Marzo de 2020, de https://www.uv.es/seoane/boletin/previos/N105-5.pdf

Oriol-Bosch, A. (2 de Junio de 2012). Scielo. Recuperado el 18 de Abril de 2020, de http://scielo.isciii.es/scielo.php?script=sci_arttext\&pid=S157518132012000200004

Palomar, J., y Gomez, N. (2010). Desarrollo de una Escala de Medición de la Resiliencia con Mexicamos (RESI-M). Dialnet, 7-22.

Puerta, M. (19 de Julio de 2017). Crianza y Salud. Obtenido de https://crianzaysalud.com.co/familia-y-resiliencia/

Truffino, J. C. (Septiembre de 2009). Resiliencia: una aproximación al concepto. (s. f. matter, Ed.) Revista de Psiquiatría y Salud Mental, 3(4), 145-151. doi:10.1016/j.rpsm.2010.09.003

UNESCO. (Febrero de 2017). UNESCO.ORG. Obtenido de https://es.unesco.org/themes/inclusion-educacion/personas-discapacitadas 
Familia como espacio de resiliencia en las personas con capacidades diferentes de la Facultad de Ciencias Humanísticas y Sociales

428

Valladáres, J. R. (17 de 03 de 2017). Enfoque en la Familia. Obtenido de

https://www.enfoquealafamilia.com/single-post/2017/05/03/La-familia-parala-persona-con-discapacidad

Vargas, V. (Junio de 22 de 2018). Resiliencia y coevolución neuroambiental. Revista Educación, 42(2). doi:https://doi.org/10.15517/revedu.v42i2.28137 